\title{
Optimized Opportunistic Routing in Space-Sky Information Network Based on Mesh
}

\author{
Zhenhao Zhang ${ }^{1}$, Nan Xiao ${ }^{1}$, Jun Liang ${ }^{1}$, Weilong Chen ${ }^{1}$, Bo Jin ${ }^{1}$ and Lingfeng Sun ${ }^{2}$ \\ ${ }^{1}$ Information and Navigation College, Air Force Engineering University, Xian, Shanxi Province, China \\ ${ }^{2}$ The $28^{\text {Th }}$ Research institute of China Electronics Technology Group Corporation, Nanjing, Jiangsu Province, China
}

\begin{abstract}
Aiming at satisfying the requirement of diversified services and insufficient calculation ability in Space-based backbone Mesh network and solving the problem of not fully considering congestion control, unreliable acknowledgement and different service in requirement of QoS guarantee in the SOAR algorithm, this paper proposed a new opportunistic routing algorithm with reliable joint acknowledge and supporting multi-service. The algorithm defined expected successful transmission count to describe the integral state of links and effectively took the load balancing and link congestion control into consideration. The new algorithm designed an adaptive route selection to support diversified service. It achieved the self-adaptation control between route selection and diversified services. Simulation results show that the algorithm in the paper has better preference in adaptively selecting the best transmission path according to the difference of service. What's more, the algorithm in the paper has better preference in the transmission delay, the throughput and the throughput rate than the SOAR routing algorithm.
\end{abstract}

Keywords-space-based backbone network; routing protocol; space-sky information network; mesh network; analytic hierarchy process

\section{INTRODUCTION}

The Space-based Mesh network, as the application of emerging broadband wireless Mesh network on the space-sky information platform, is capable of efficiently enlarging the coverage are of the wireless Mesh network. The routing protocol, one of the vital fields studied by the Space-based Mesh network, has become the focus of present researches of the Space-based Mesh network. According to the document [1], researches about opportunistic routing protocol conducted by the wireless Mesh network has become the hotspot in the field of routing protocol study. The ExOR opportunistic routing protocol put forward by the document [2], but the routing algorithm lacks the balancing control of link load and suffers from comparatively low throughput. The document [3] leading to the low efficiency of forwarding packets of forwarding nodes selected by the routing path. The document [4] neglects the caching capability of forwarding nodes and this algorithm is very likely to produce congestion of the optimization path and lowers the forwarding efficiency of nodes. The SOAR protocol proposed by the document [5], but this algorithm fails to take the influence placed by the caching and forwarding capability of middle transmission nodes on the reception of data packets and link throughput into account. Moreover, this algorithm can't choose the optimization transmission link in accordance with demands of QoS made by the transmission of different service.

In terms of the above problems existing in the early research of the opportunistic routing and algorithm of traditional SOAR routing, this paper comprehensively considers the delivery rate of nodes, the caching size of transmission nodes and the size of available transmission space and puts forward the optimized SOAR routing algorithm featuring high reliability and multi-service transmission.

\section{NETWORK MODELS}

The Space-based backbone Mesh network is flexibly organized and constructed by low earth orbits featuring stable connection, low relative motion and high reliability, near-space low dynamic aircrafts and large-scale air backbone platforms through broadband data chains [6]. The user nodes (such as fighter, guided missiles and high-dynamic aircrafts and so on) could realize the high-speed transmission and interaction of information in wide range by accessing the Space-based Mesh network. The network topology diagram is as follows:

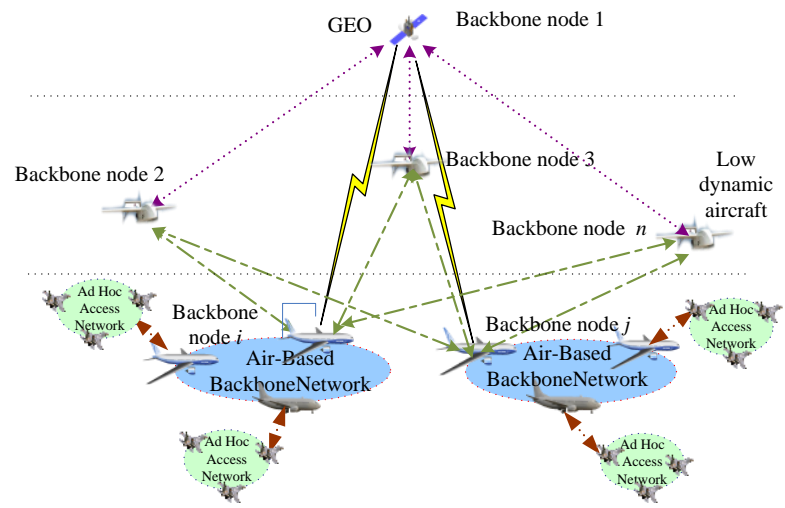

FIGURE I. THE DIAGRAM OF ROUTING AND NODES FORWARDING

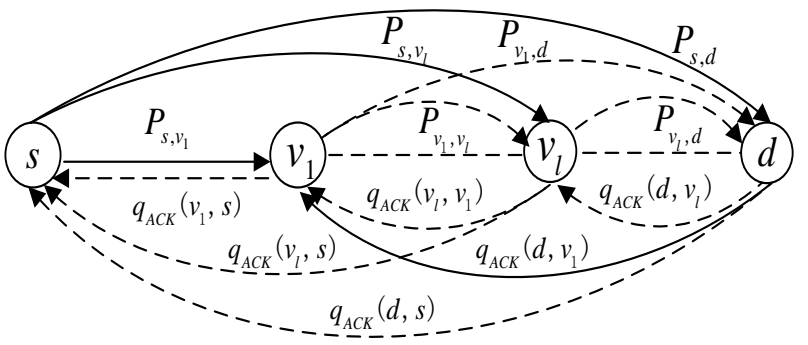

FIGURE II. THE MODEL OF ROUTING AND NODES FORWARDING

\section{SOAR ROUTING MODEL}

\section{A. Model Construction}

In view of the model of in Figure 1, the digraph $G=(V, L$, 
$D$ ) could be used. $V$ expresses the assemblage of nodes in Mesh network and includes the node $v_{l} ; D$ indicates the delivery rate of nodes and the delivery rate between any two nodes $\left(v_{m}, v_{l}\right)$ is $d_{m, l} \in D$. The delivery rate of nodes when receive and transmit data packets is $1^{[2]}$.It's assumed that $s, d \in V$ act as the sending node and receiving node of data packets. In the network, there are $n+1$ nodes to construct the retransmission list $F_{s, d}=\left\{s=v_{0}, v_{1}, v_{2}, \cdots, v_{n}=d\right\}$. The subscript of node should be enlarged and the priority should be improved ${ }^{[7]}$. According to the above definition, the model of routing and nodes forwarding in the whole process of data packet transmission is constructed in Figure 2.

Where $q_{A C K}\left(v_{l}, v_{m}\right)$ indicates the ACK delivery rate between $v_{l}$ and $v_{m} ; P_{v_{m}, v_{l}}$ expresses the probability that data packets sent by the retransmission node $v_{m}$ in the retransmission list $F_{s, d}$ are received by the node $v_{l}(m<l)$ whose priority is higher than that of $v_{m}$ but not received by the node $v_{j}(l<j)$ whose priority is higher than that of $v_{l}$. The calculation could be conducted because links are traditionally independent.

$$
P_{v_{m}, v_{l}}= \begin{cases}d_{v_{m}, v_{l}} \prod_{j=l+1}^{n}\left(1-d_{v_{m}, v_{j}}\right) & \forall m \leq l \\ 0 & \forall m>l\end{cases}
$$

\section{B. Routing Metrics}

The traditional SOAR routing protocols employ ETX as the method to measure routing to find the optimization transmission path. In terms of the retransmission list $F_{s, d}=\left\{s=v_{0}, v_{1}, v_{2}, \cdots, v_{n}=d\right\} \quad{ }^{[9]}, \quad$ the expected transmission number can be expressed in the formula (2).

$$
\operatorname{ETX}_{F_{s \Leftrightarrow d}}=R_{v_{0}, v_{n}}=\frac{\left(1+\sum_{l=1}^{n}\left(P_{v_{0}, v_{l}} \cdot q_{A C K}\left(v_{l}, v_{0}\right)\right) \cdot R_{v_{l}, v_{n}}\right)}{\sum_{l=1}^{n}\left(P_{v_{0}, v_{l}} \cdot q_{A C K}\left(v_{l}, v_{0}\right)\right)}
$$

\section{THE OPPORTUNiSTIC ROUTING PROTOCOL SUPPORTING SPACE-BASED MESH NETWORK}

\section{A. The Design of Reliable Response Mechanism}

In view of features like long time delay of transmission of satellite nodes in the Space-based backbone Mesh network and highly reliable data transmission needed by air nodes, the traditional ACK mechanism should be improved to effectively reduce the retransmitted data packets which are caused by the ACK response in the Space-based backbone Mesh network. This paper uses the Reliable Joint Acknowledge to integrate the ACK response with SNACK to make it qualified to be used in the Space-based backbone
Mesh network. The reversed ACK delivery rate between any two nodes is $q_{A C K}\left(v_{l}, v_{m}\right)$, and set the transmission time of RJACK as: $i=\left[1 / q_{A C K}\left(v_{l}, v_{m}\right)\right]+1$.At this moment, the delivery rate of RJACK could be expressed by the formula (3):

$$
q_{R J A C K}=1-\left(1-q_{A C K}\left(v_{l}, v_{m}\right)\right)^{i}
$$

\section{B. The Optimization of Routing Metrics}

SOAR routing protocol uses the end-to-end delivery rate to measure the probability, but whether data packets could be smoothly transmitted to the next node is totally based on the caching and transmission capability of nodes. Meanwhile, the link congestion control will greatly affect the transmission efficiency of network. For this reason, the successful retransmission rate is proposed in this paper. The successful delivery rate is based on the delivery rate $d_{x, y}$ between two nodes, the size of cache region of next node and the available size of transmission space. The normalization coefficient of cache region of any node 1 could be expressed as: $\alpha=T R_{l} / R c R_{l}^{2}$. On the basis of it, the successful delivery rate $Z_{x, y}$ could be expressed by the formula (4):

$$
Z_{x, y}=\alpha \times(1-\beta) \times d_{x, y}
$$

In view of the Space-based Mesh network, the successful delivery rate is regarded as new measurement index, and expected successful transmission count is put forward to describe the performance of whole link. The congestion control and load balancing could be better realized by building minimum ESTX link and regarding it as the optimization link to transmit large data-volume service. The method to set threshold value is similar to that to set ETX threshold value in SOAR routing algorithm [5].

On the basis of the above description of ESTX, define $Q_{v_{m}, v_{l}}$ which expresses the probability that data packets sent by the retransmission node $v_{m}$ in the retransmission list $F_{s, d}$ successfully cached and retransmitted by the node $v_{l}(m<l)$ whose priority is higher than that of $v_{m}$ but not successfully cached and retransmitted by the node $v_{j}(l<j)$ whose priority is higher than that of $v_{l}$. The $Q_{v_{m}, v_{l}}$ could be expressed by the formula (5).

$$
Q_{v_{m}, v_{l}}= \begin{cases}Z_{v_{m}, v_{l}} \prod_{j=l+1}^{n}\left(1-Z_{v_{m}, v_{j}}\right) & \forall m \leq l \\ 0 & \forall m>l\end{cases}
$$

Regard the successful delivery rate $Z_{x, y}$ as the new delivery rate of improved SOAR routing protocol and the ESTX calculation formula expressed by the formula (6) could be obtained based on the formula (2). $R_{v_{l}, v_{n}}^{\prime}$ indicates the 
expected successful transmission count of data transmitted from any middle retransmission node $v_{l}$ to the target node $v_{n}$

$$
\operatorname{ESTX}_{F_{s \oplus d}}=R_{v_{0}, v_{n}}^{\prime}=\frac{\left(1+\sum_{l=1}^{n}\left(Q_{v_{0}, v_{l}} \cdot q_{A C K}\left(v_{l}, v_{0}\right)\right) \cdot R_{v_{l}, v_{n}}^{\prime}\right)}{\sum_{l=1}^{n}\left(Q_{v_{0}, v_{l}} \cdot q_{A C K}\left(v_{l}, v_{0}\right)\right)}
$$

\section{Dynamic Path Selection}

The Space-based Mesh network, as the backbone network, should provide users with diversified high-reliability and high-quality pictures and videos and some other multimedia service and the diversified service always puts relevant demands on the quality of service. The time delay, bandwidth, packet loss probability and throughput demanded by different service should be comprehensively considered when the optimization link is confirmed in routing protocols because users tend to have different demands towards relevant service in view of the service provided by the Space-based Mesh network. This paper uses the AHP to get weight definition on the basis of the type of service. Therefore, the AHP model is constructed as figure 3 .

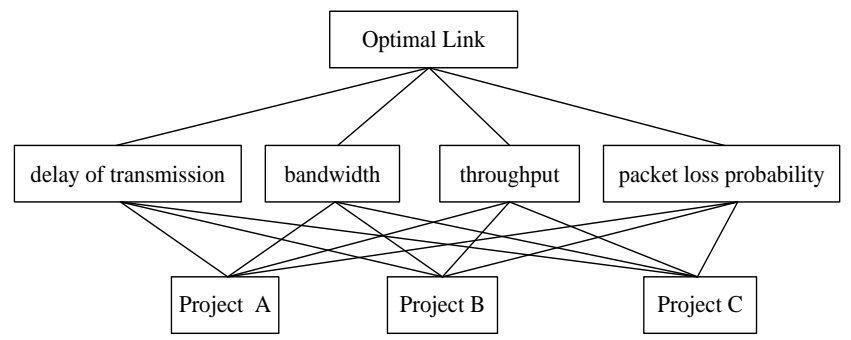

FIGURE III. CONSTRUCTION OF AHP MODEL

In accordance with the AHP model constructed to calculate the weight of all evaluation parameters in the Figure 3 and in view of different transmission service in networks, different weight coefficients are set on the basis of the above four evaluation parameters $\lambda_{1}, \lambda_{2}, \lambda_{3}, \lambda_{4}$. Use the utility function $\Gamma=\lambda_{1} \omega_{1}+\lambda_{2} \omega_{2}+\lambda_{3} \omega_{3}+\lambda_{4} \omega_{4}$ to confirm the optimization routing path, where $\omega_{1}, \omega_{2}, \omega_{3}, \omega_{4}$ respectively indicates four parameters (time delay, throughput, packet loss probability and network load) which are needed to be considered by the AHP model when it selects the optimization path.

\section{SimULATION AND ANALYSIS}

\section{A. Model of Network Transmission Service}

Air-space information network integrates numerous air-based and space-based communication platforms. The transmission service provided by the Space-based backbone Mesh network could be divided into three parts in accordance with demands of network transmission service on different indicators: SMS service (command information, situation information, etc.), dialogue service (voice service) and multimedia service (videos, pictures, etc.). On the basis of the demands of different service on QoS, the weights of three service towards four evaluation parameters could be obtained, which is shown in the Table 1.

TABLE I. THE RELATIONSHIP BETWEEN WEIGHT OF EVALUATION PARAMETERS AND TYPES OF SERVICES

\begin{tabular}{|c|c|c|c|c|}
\hline \multirow{2}{*}{$\begin{array}{c}\text { types of } \\
\text { services }\end{array}$} & \multicolumn{4}{|c|}{ Weight of Evaluation Parameters } \\
\cline { 2 - 5 } & $\begin{array}{c}\text { Delay of } \\
\text { transmissio } \\
\mathrm{n}\end{array}$ & $\begin{array}{c}\text { Throughpu } \\
\mathrm{t}\end{array}$ & $\begin{array}{c}\text { Bandwi } \\
\mathrm{dth}\end{array}$ & $\begin{array}{c}\text { delay of } \\
\text { transmissio } \\
\mathrm{n}\end{array}$ \\
\hline SMS service & 0.2660 & 0.0841 & 0.0532 & 0.5967 \\
\hline $\begin{array}{c}\text { dialogue } \\
\text { service }\end{array}$ & 0.5926 & 0.1508 & 0.1365 & 0.1201 \\
\hline $\begin{array}{c}\text { multimedia } \\
\text { service }\end{array}$ & 0.2356 & 0.2764 & 0.2897 & 0.1983 \\
\hline
\end{tabular}

\section{B. Simulation and Verification}

\section{1) Performance Comparison of Routing Protocols}

It is assumed that the space-based backbone Mesh network consists of three large airborne platforms, two near-space vehicles and a satellite. In the paper, it sets up the load intensity of network transmission, which is divided into three grades including low, medium and high grade assigned that the normalized value of $\mathrm{L}=0.2,0.5$ and 0.8 .The sending and receiving packets of the source node and the destination node are not limited by the cache size, and the other forwarding nodes are randomly set up with three groups of different cache size. The nodes in the simulation are randomly distributed in a square area, and the distance between nodes and its neighbors is no more than $40 \mathrm{~km}$. which can be set up to 10000 times. The number of simulation in this paper is set to 10000 times.

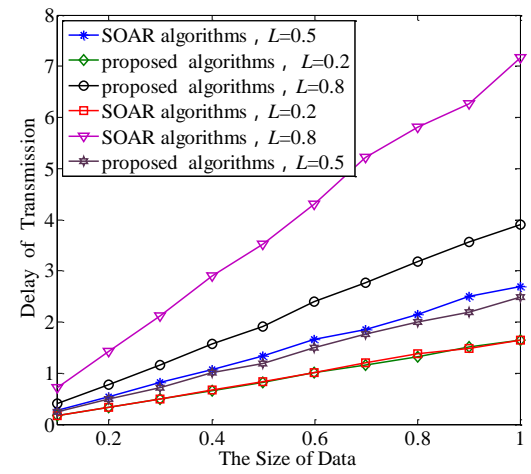

FIGURE IV. THE RELATIONSHIP BETWEEN THE SIZE OF DATA AND TRANSMISSION DELAY

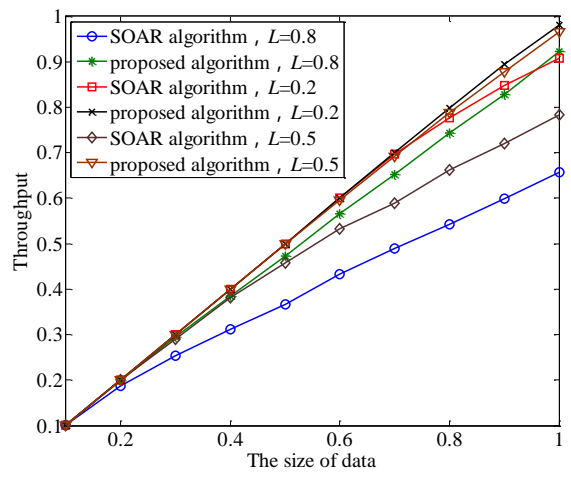

FIGURE V. THE RELATIONSHIP BETWEEN THE SIZE OF DATA AND THROUGHPUT 


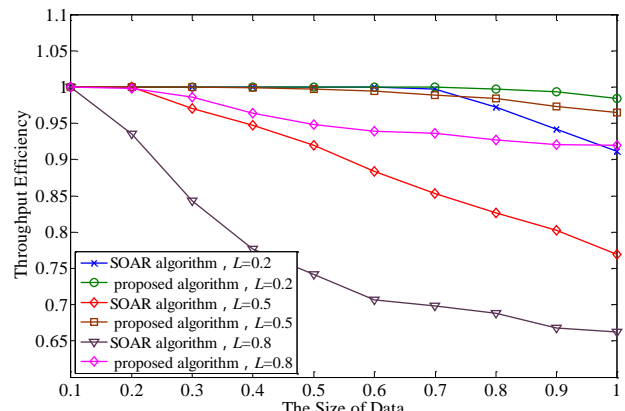

FIGURE VI. THE RELATIONSHIP BETWEEN THE SIZE OF DATA AND THROUGHPUT EFFICIENCY

Simulation results in Figure 7 to 9 indicate that the proposed routing algorithm compared with the traditional SOAR routing algorithm has improvement in performance of transmission delay, throughput and throughput efficiency. On one hand, the routing algorithm in this paper has the same performance as that of traditional SOAR routing algorithm in condition of small data size and light load. On the other hand, the proposed routing algorithm has better performance than that of traditional SOAR routing algorithm in condition of big data size and heavy load.

\section{2) Performance Comparison of Routing Protocols on} Diverse Traffic Patterns

Comparison in part A can proof that the routing algorithm in this paper has better performance than that of traditional SOAR routing algorithm. In this part, the simulation environment is set to the same as that in part A. The simulation is to compare the performance of two routing algorithms in condition of different traffic types and different load intensity. The simulation results are shown in Figure 10 and Figure 11.

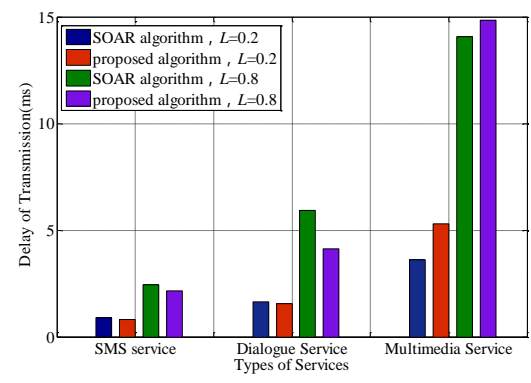

FIGURE VII. THE RELATIONSHIP BETWEEN THE DIFFERENT TYPE OF SERVICE AND TRANSMISSION DELAY IN DIFFERENT NETWORK LOAD

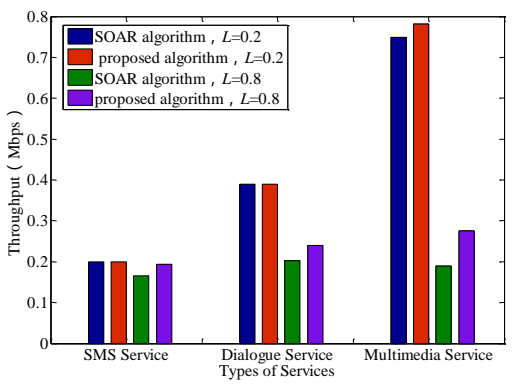

FIGURE VIII. THE RELATIONSHIP BETWEEN THE DIFFERENT TYPE OF SERVICE AND THROUGHPUT IN DIFFERENT NETWORK LOAD
The simulation result shown in Figure10 to 11 illustrates that the throughput performance of the proposed routing algorithm is significantly improved compared with traditional SOAR routing algorithm in the case of transmitting multimedia service, but the transmission delay is increased. In the case of transmitting small traffic service such as short message service, the transmission delay and throughput performance are not improved obviously. When the session service is transmitted, the performance of transmission delay and throughput is significantly improved.

\section{REFERENCES}

[1] Alotaibi, Eiman, and B. Mukherjee. "A survey on routing algorithms for wireless Ad-Hoc and mesh networks." Computer Networks 56.2(2012):940-965.

[2] Biswas, Sanjit, and R. Morris. "ExOR: opportunistic multi-hop routing for wireless networks." Conference on Applications, Technologies, Architectures, and Protocols for Computer Communications ACM, 2005:133-144.

[3] LI Feng, SHEN Song. "The Study of Opportunistic Routing for Wireless Mesh Networks". Electronic Design Engineering, 24.5(2016):58-61

[4] WANG Dayu, ZHANG Shaohui, SONG Yufei. "Coordinated Opportunistic Routing Protocol with Single-rate Using ETX Routing Metric in WMN".Application Research of Computers, 33.5 (2016): 1468-1472.

[5] Rozner E, Seshadri J, MethaY A, et al. SOAR: Simple Opportunistic Adaptive Routing Protocol for Wireless Mesh Networks [J]. IEEE Transactions on Mobile Computing, 2009, 8(12): 1622-1635.

[6] GE Xiaohu, LIU Yingzhuang, DONG Yan, et al. "A Space-Sky Information Network Model Based on MESH Architecture". Microelectronic \& Computer, 25.5 (2008):39-42.

[7] K.Miyakita, K Nakano, M. Sengoku, and S.Shinoda. Theoretical analysis of routing expected transmission count in multi-hop wireless networks [J].IEICE Transactions on Communications, 2010, 91(8): 2533-2544.

[8] ZHAO Chuanqiang, Lin Yuanan, LIU Kaiming, et al. "A Throughput Efficiency Oriented Opportunistic Routing in Wireless Mesh Networks". Journal of Beijing University of Posts and Telecommunications, 34.2 (2011):81-84.

[9] Cacciapuoti, A. S, M. Caleffi, and L. Paura. "A theoretical model for opportunistic routing in ad hoc networks." International Conference on Ultra Modern Telecommunications, Icumt 2009, 12-14 October 2009, St. Petersburg, Russia DBLP, 2009:1-7.

[10] CHENG Jie, ZHANG Xizhi. "Simulation of Sensor Network Algorithm Based on Dynamic Weight". Computer Simulation, 32.4 (2015):81-84

[11] Tao, W., L. Wenwei, and H. Shiming. "Node load balance multi-flow opportunistic routing in wireless mesh networks." Sensors \& Transducers 168.4(2014):162-172. 